\title{
Why not record from every channel with a CMOS scanning probe?
}

George Dimitriadis ${ }^{\dagger}, 1, *$, Joana P. Neto ${ }^{1,8,18, *}$, Arno Aarts ${ }^{2}$, Andrei Alexandru ${ }^{3}$, Marco Ballini ${ }^{3}$, Francesco Battaglia ${ }^{4}$, Lorenza Calcaterra ${ }^{1}$, Francois David ${ }^{5}$, Richárd Fiáth ${ }^{6,7}$, João Frazão ${ }^{8}$, Jesse $\mathrm{P}$ Geerts $^{1}$, Luc J. Gentet ${ }^{5}$, Nick Van Helleputte ${ }^{3}$, Tobias Holzhammer ${ }^{2}$, Chris van Hoof ${ }^{3}$, Domonkos Horváth $^{6,7,9}$, Gonçalo Lopes ${ }^{1}$, Carolina M. Lopez ${ }^{3}$, Eric Maris ${ }^{4}$, Andre Marques-Smith ${ }^{1}$, Gergely Márton $^{6,7}$, Bruce L. McNaughton ${ }^{19,20}$, Domokos Meszéna ${ }^{6,7}$, Srinjoy Mitra ${ }^{10}$, Silke Musa ${ }^{3}$, Hercules Neves $^{11,17}$, Joana Nogueira ${ }^{1}$, Guy A. Orban ${ }^{12}$, Frederick Pothof ${ }^{13}$, Jan Putzeys ${ }^{3}$, Bogdan Raducanu ${ }^{3,14}$, Patrick Ruther ${ }^{13}$, Tim Schroeder ${ }^{4}$, Wolf Singer ${ }^{15}$, Paul Tiesinga ${ }^{16}$, Istvan Ulbert ${ }^{6,7}$, Shiwei Wang ${ }^{3}$, Marleen Welkenhuysen ${ }^{3}$, Adam R. Kampff ${ }^{1}$

†g.dimitriadis@ucl.ac.uk

* These authors contributed equally to this work

1) Sainsbury Wellcome Centre for Neural Circuits and Behaviour, University College London, United Kingdom

2) ATLAS Neuroengineering, Leuven, Belgium

3) imec, 3001 Leuven, Belgium

4) Donders Institute for Brain, Cognition and Behavior, Radboud University Nijmegen, The Netherlands

5) Team Waking, Lyon Neuroscience Research Center (CNRL), INSERM-U1028, CNRS-UMR5292, Bron, France

6) Institute of Cognitive Neuroscience and Psychology, Research Centre for Natural Sciences, Hungarian Academy of Sciences, Budapest, Hungary

7) Faculty of Information Technology and Bionics, Pazmany Peter Catholic University, Budapest, Hungary

8) Champalimaud Neuroscience Programme, Champalimaud Centre for the Unknown, Portugal

9) School of PhD Studies, Semmelweis University, Budapest, Hungary

10) Institute for Integrated Micro and Nano Systems (IMNS), University of Edinburgh, UK

11) Uppsala University, Sweden

12) Department of Medicine and Surgery, University of Parma, Parma, Italy

13) Department of Microsystems Engineering (IMTEK), University of Freiburg, Germany

14) Electrical Engineering Department-ESAT, KU Leuven, 3001 Leuven, Belgium

15) ErnstStrüngmann Institute for Neuroscience in Cooperation with Max Planck

16) Neurinformatics department, Donders Institute for Brain, Cognition and Behavior, Radboud University Nijmegen, Heyendaalseweg 135, 6525 AJ, Nijmegen, The Netherlands

17) Fiocruz, Brazil

18) Departamento de Ciência dos Materiais, CENIMAT/I3N and CEMOP/Uninova, Faculdade de Ciências Tecnologia-Universidade Nova de Lisboa, Caparica, Portugal

19) Department of Neurobiology and Behavior, University of California at Irvine, Irvine CA, USA, 92697-8439

20) Department of Neuroscience, The University of Lethbridge, Lethbridge, AB, Canada, T1K $3 M 4$ 
bioRxiv preprint doi: https://doi.org/10.1101/275818; this version posted February 9, 2019. The copyright holder for this preprint (which was not certified by peer review) is the author/funder, who has granted bioRxiv a license to display the preprint in perpetuity. It is made available under aCC-BY 4.0 International license. 


\section{Abstract}

Neural recording devices normally require one output connection per electrode. This limits the number of electrodes that can fit along the thin shafts of implantable probes. Sharing a single output connection between multiple electrodes relaxes this constraint and permits designs of ultra-high density probes.

Here we report the design and in-vivo validation of such a device, a complementary metal-oxidesemiconductor (CMOS) scanning probe with 1344 electrodes along an $8.1 \times 0.1 \times 0.05 \mathrm{~mm}$ shaft; the outcome of the European research project NeuroSeeker. Addressing the challenges of data management and visualization, we present novel methods for monitoring recordings. We also describe chronic implantation results and a method to extract and reuse the device over multiple animals.

Scanning CMOS technology allows much smaller, denser electrode arrays. To help design electrode configurations for future probes, recordings from many different brain regions were obtained with an ultra-dense passive probe.

All datasets are available online.

\section{Introduction}

The number of neurons that can be monitored simultaneously during an extracellular recording is currently limited by the number of electrodes that can be implanted (Stevenson, 2017; Stevenson and Kording, 2011). However, the desire for large-scale monitoring of neural activity and the need to minimize tissue damage compete with one another (Buzsáki et al., 2015). The high resolution processes of semiconductor fabrication (i.e., photolithographic patterning of thin film conductors and insulators on a silicon substrate) have enabled dozens of microelectrodes, packed with an ever increasing density, along needle-like probes (Berényi et al., 2014; Blanche, 2005; Buzsáki et al., 2015; Du et al., 2011; Shobe et al., 2015; Torfs et al., 2011). In the standard "passive" silicon probe, electrodes distributed along the probe shaft must each be connected to an external contact pad on the probe base by metal lanes deposited along the shaft. Advances in lithography techniques (i.e., ebeam lithography (Rios et al., 2016; Scholten and Meng, 2016)), metal deposition procedures, and quality control have made it possible to fabricate a five shank, 1000-channel probe, where each shank (width of $\sim 50 \mu \mathrm{m}$ ) has 200 electrodes of $9 \times 9 \mu \mathrm{m}$ and a pitch of $11 \mu \mathrm{m}$ (Scholvin et al., 2016). Despite these impressive designs, a bottleneck remains in the number of output connection lanes that can be squeezed into a single thin probe shaft. Therefore, monitoring the thousands of electrodes required to densely cover an $8 \mathrm{~mm} \times 100 \mu \mathrm{m}$ probe will demand a new technology; a technology that multiplexes the signal from multiple electrodes into one output lane.

The semiconductor industry has solved the same interconnect challenge with integrated circuitry (IC), often fabricated using the CMOS manufacturing process. As a characteristic example, mobile phones with on-board cameras use CMOS image sensors (CIS) that are lightweight and low power, but that can convert an optical image into an electronic signal with very high spatial resolution. A CIS is composed of an array of millions of identical pixels, each having at least a photodiode, an addressing transistor that acts as a switch, and an amplifier. Each pixel is sampled once within each frame and the image output is generated by rapidly scanning the full sensor matrix. This active switching / time multiplexing is crucial for achieving the small size of a CIS device, as it conveys the signals from many pixels with just a few output connections. 
In the case of silicon probes, their one-dimensional architecture, high sampling speed requirements ( 3 orders of magnitude larger than a CIS), the low amplitude of the neural signals (three orders of magnitude lower than the photodiode signals in CMOS cameras) and the need to avoid tissue overheating due to electrical power dissipation have challenged the inclusion of integrated circuitry within the probe's shaft. The concept of integrating electronic elements in the same substrate used to build the recording electrodes was first introduced in the 1980s (Najafi and Wise, 1986; Najafi et al., 1985; Wise and Ji, 1992; Wise and Najafi, 1991) The most recent CMOS-based implantable probe designs for in vivo applications integrate circuitry into both the probe base and within the probe shaft(s) and have been reviewed elsewhere (Lopez et al., 2013, 2016; Obien et al., 2015; Raducanu et al., 2016; Ruther and Paul, 2015; Seymour et al., 2017; Steinmetz et al., 2018).

The NeuroSeeker European research project aimed to produce CMOS-based, time multiplexing, silicon probes for extracellular electrophysiological recordings with 1344 electrodes on a $50 \mu \mathrm{m}$ thick, $100 \mu \mathrm{m}$ wide, and $8 \mathrm{~mm}$ long shank with a $20 \times 20 \mu \mathrm{m}$ electrode size and a pitch of $22.5 \mu \mathrm{m}$ (see Box 1 for a technical overview of the probe and (Raducanu et al., 2016, 2017) for an in depth description of the probe's design). This innovative solution has allowed the neuroscientists within the consortium to record brain activity with an unprecedented combination of resolution and scale. Here we report the use of the NeuroSeeker probe to record from both anesthetized and freely moving rats. We characterize in saline and in vivo the increase in noise that the local amplification and time multiplexing scheme incurs relative to a passive electrode probe with the same configuration. We show that this increase is insignificant in vivo and does not inhibit the capture of high quality neural signals. In the case of freely moving animals, we also include data showing that the signal remains stable for at least 10 days. We also describe a method for removing the NeuroSeeker probe intact, and ready for reuse, from the brain of a chronically implanted animal and show results from freely moving recordings from an animal implanted with such a 'second hand' probe. This allows the multiple re-use of probes whose price, and low production numbers, makes them currently inaccessible to all but a tiny minority of electrophysiology labs around the world.

Recording with this number of electrodes results in datasets of around $200 \mathrm{~GB}$ per hour with spike numbers in the millions. This scale of data poses new difficulties in the visualization, pre-processing, and curation of the signal, even before it can be used to explore correlations with sensory stimuli or behaviour. Here we present an online visualization method that allows for the rapid overview of the signal from all 1344 electrodes, giving the experimenter a global picture of the recording quality as data is acquired.

Given that CMOS probes with local amplification and time multiplexing allow a significant increase in electrode density without sacrificing compactness, the neuroscientific community must now determine the physiologically relevant limit for the size and density of the electrodes on such probes. To help address this question, we have collected datasets from ultra-high density (256 electrodes of $5 \times 5 \mu \mathrm{m}$ size with a $6 \mu \mathrm{m}$ pitch) passive probes. These were recorded from anesthetized rats in a wide range of brain structures and probe orientations. These datasets are available online for further analysis of the optimal geometries for future CMOS probes. 


\section{Results}

Several recordings were performed with the NeuroSeeker CMOS-based scanning probes in vivo to evaluate the viability of these devices. There are 12 groups of electrodes on the probe, which can be individually powered, labelled from $\mathrm{G} 1$ to $\mathrm{G} 12$, in Box 1 and Figure 1. Each group contains 120 channels: 112 electrodes and 1 local reference in the middle made from a group of 8 electrodes binned together. For each electrode, the user can select the reference type (external or internal), gain, and frequency bandwidth (high-pass cut-off frequency in AP mode or low-pass cut-off frequency in LFP mode). For all recordings, 1277 electrodes were set in the AP band $(0.5-7.5 \mathrm{kHz})$, while 67 were set in the LFP band $(1-500 \mathrm{~Hz})$. The LFP electrodes were spaced 20 electrodes apart from one another, which translates to 5 rows, or, given the $22.5 \mu \mathrm{m}$ pitch of each electrode, 112.5 $\mu \mathrm{m}$.

\section{Neural recordings Acute recordings}

Figure $1 \mathrm{~A}$ shows a representative epoch of a recording performed simultaneously within cortex, hippocampus, and thalamus from an anesthetized rat. In this recording, 10 of the 12 groups are in the brain and thus enabled (powered on): 1060 out of the 1120 electrodes are set in AP mode with 60 set to LFP mode. A subset of traces from electrodes set in AP and LFP mode are shown in Figure $1 \mathrm{~A}$ (a recording segment of all traces is shown in Supplementary Figure 1). The traces in AP mode are displayed in groups ( $c 1, c 2, c 3, c g, h 1, h 2, h 3, h 4, t 1, t 2, t 3$ and t4) that correspond to the location on the probe shaft indicated by the black region in the probe schematic. Moreover, traces of 11 out of 60 LFP mode electrodes are plotted between the group traces. The presence of fast voltage deflections in several traces reveals that the spiking activity of multiple neurons has been detected. Furthermore, the NeuroSeeker probe densely samples the local electric field, providing a detailed description of the spatiotemporal profile of a neuron's extracellular action potential, as shown in Figure 1C.

To evaluate if the data quality deteriorates with increasing number of groups powered, we computed the noise of 212 electrodes (from groups G1 and G2) set in AP mode while enabling more groups along the shank. The noise level for the 212 electrodes using the external reference, with 2 groups and 12 groups powered, is $10.2 \pm 0.1 \mu \mathrm{V}$ and $12.0 \pm 0.1 \mu \mathrm{V}$, respectively. Additionally, the noise magnitude was computed for the 1012 channels in the brain (half of group 10's electrodes are outside of the brain) using either the external or the internal reference configuration while recording. The measured noise using the external and internal reference is $11.7 \pm 0.1 \mu \mathrm{V}$ and $12.5 \pm$ $0.1 \mu \mathrm{V}$, respectively. The noise magnitude computed across these datasets revealed no significant variability. We also measured the noise in saline for the NeuroSeeker probe. The measured noise in saline while powering the same 1012 channels was $9.4 \pm 0.1 \mu \mathrm{V}$. We compared the noise measured in saline and during acute recordings with a 128-channel probe previously described (Neto et al., 2016). This passive electrode probe has the same configuration, electrode size, and electrode material (titanium nitride) as the CMOS based probe (impedance magnitude at $1 \mathrm{kHz}$ is around 50 $\mathrm{k} \Omega$ ). The noise value calculated with the 128 -channel probe in saline was $2.69 \pm 0.02 \mu \mathrm{V}$ and in vivo (amplifier2015-08-28T20_15_45 in http://www.kampff-lab.org/validating-electrodes/) was $10.40 \pm$ $0.04 \mu \mathrm{V}$. Despite the difference in saline (9.4 vs $2.7 \mu \mathrm{V}$ ) the noise difference in vivo was small (11.7 vs $10.4 \mu \mathrm{V}$ ) between the CMOS based probe and the passive 128-channel probe, highlighting the dominance of "biological" noise for in vivo extracellular recordings.

Several recordings were performed with different reference configurations and number of active groups. These datasets are available online (http://www.kampff-lab.org/cmos-scanning/) and 
summarized in Supplementary Table 1. Figure 1 is derived from one of these recordings, 18_26_30.bin.

\section{Chronic recordings}

We also conducted a set of recordings from chronically implanted animals. Given the high cost and low availability of high density probes, we designed a method that allowed us to extract the CMOS probe from the brain of a chronically implanted animal at the end of the experimental period so that it can be reused for multiple experiments. This required a specially designed probe holder and a series of steps after animal euthanasia to ensure the probe is removed without breaking the fragile shaft. The holder is a two-part design and is shown in Figure 2A, while in Methods we describe a detailed probe recovery technique. We have managed to successfully remove 4 out 5 chronically implanted probes (two dummy and two fully functional). In the case of the unsuccessful attempt, the probe holder exhibited a small angle deflection off the Dorsal-Ventral / Anterior-Posterior plane, even before the cutting of the resin, indicating that the probe had already moved and thus broken while the animal was alive.

We present here recordings from two animals, both implanted with the same probe. In both cases the probe was fully implanted in the brain targeting the parietal cortex and the subcortical regions beneath (see Figure $2 \mathrm{~B}$ schematics for a description of the anatomical regions the probe was passing through), thus recording from 1277 electrodes from the AP band and 67 electrodes from the LFP band. In both cases, the probe was left in the animal for 4 weeks. Each animal was recorded daily in a freely moving session which ranged between 40 to 70 minutes. Each session generated between 1.5 and 3.5M spikes. We spike sorted some representative sessions to demonstrate the robustness of the recordings over time and our ability to remove the probe from one animal, implant it in another, and still get recordings of the same quality as a new probe. During the spike sorting pipeline (using kilosort (Pachitariu et al., 2016) and t-SNE (Dimitriadis et al., 2016)) we separated the units into 4 categories. These were the single units, i.e. groups of spikes that obviously came from the same cell (given their autocorrelogram, spike shape, and signal's position/spread on the probe), the contaminated single units (those that also had a few spikes not coming from the same cell), the putative single units (those that didn't fire enough spikes to create an autocorrelogram convincing of their single unit nature) and the multi units (the groups of spikes that obviously belonged to multiple units). Figure 2B shows the position of these units (color coded according to their assignment) on the probe shaft (see Methods on how these positions are calculated). For the first implanted rat we show three recordings, taken 3,7 and 10 days after surgery. For the second rat we show the first recording taken 3 days after surgery. Figure $2 \mathrm{C}$ shows the number of the different types of units over the different recordings. Table 1 summarizes the number of units and number of spikes recorded in each session. The number of detected templates (most of them single units) ranged between 1113 and 633. In (Dimitriadis et al., 2016) we report that in an under anesthesia session (with a different NeuroSeeker probe) and with 908 electrodes in the brain we detected 579 units, comparable to the unit count we get in these awake experiments. The data show that the probe proved to be a robust recording device, able to detect over 1000 units while not showing a significantly worse deterioration of its capacity compared to any other silicon probe over extended periods of recording.

The data also show that multiple use of the same device is possible and does not impact on the quality of the recordings. We note here that the first animal implantation was done in a slow manner (over 1 hour for the whole $8 \mathrm{~mm}$ length) while the implantation of the second animal was done in a 
fast manner ( 5 minutes for the whole probe). This might be one reason that the second animal recordings show a reduced number of neurons, especially of the clean single unit type (i.e. the neurons closer to the shaft) and of the number of spikes recorded.

\section{Data visualization}

The signal traces presented in Figure 1 are just a small fraction of the total number of electrodes (105 of 1060 electrodes) recorded during an acute experiment (see all voltage traces in Supplementary Figure 1). These ultra-high channel count CMOS scanning probes presented a new challenge for the visualization and analysis of the acquired data. This challenge first appears when the user wants to monitor an ongoing recording, either to assess the signal quality appearing on each electrode or to better position the probe within the desired brain structure(s). Visualizing 1344 timeseries is difficult, even on an HD monitor with 1080 rows of pixels. Furthermore, rendering voltage vs. time traces, the conventional representation for online physiology signals, is computationally expensive. However, modern graphic processors (GPUs) were specifically designed to parallelize such visualization tasks. We therefore developed a custom visualization pipeline using Bonsai (Lopes et al., 2015) by transferring each buffer of acquired probe data directly to the GPU and then using fragment shaders to render all probe data in real-time. The visualization shown in Figure 3 is a screen capture of one such online visualization in which the voltage signal of each channel is rendered from red to blue (also see Supplementary movie 1). A neuron's extracellular action potential is detected on many adjacent electrodes, and thus spikes in this visualization appear as a coloured block of red and blue stripes.

\section{Do we need all these electrodes?}

Integrating CMOS-based scanning technology into the shaft of an in vivo neural probe has now made it possible to drastically increase electrode density. However, given the challenges associated with the volume of data generated by such probes, neuroscientists must decide whether further increases in recording density are actually useful. Current approaches to spike sorting would appear to benefit from higher density probes (Dimitriadis et al., 2016; Moore-Kochlacs, 2016; Rossant et al., 2015). Furthermore, initial results (Jun et al., 2017a) show that increased electrode densities could help compensate for drift in chronic recordings and allow following individual neurons over the course of days and weeks. Finally, CMOS technology enables the fabrication of electrodes much smaller than a neuron's soma and dense enough to capture fine details of a single neuron's extracellular field throughout its dendritic tree (Delgado Ruz and Schultz, 2014; Jia et al., 2018).

Whether useful information is present at these scales, and whether it can contribute to further understanding of brain function, is an open question. Thus, in order to address whether ultra-high density in neural recordings will be useful, we collected a series of datasets with an ultra-dense probe during anesthesia from many different brain regions (Figure 4).

The ultra-dense probe contains 255 electrodes (and one large reference) with a geometric area of 5 x $5 \mu \mathrm{m}$ and separated by $1 \mu \mathrm{m}$, and was fabricated by IMEC as part of the NeuroSeeker project. The goal of these experiments was to understand at what spatial scale the signals from neighbouring electrodes became redundant (i.e., the extracellular signal becomes spatially over-sampled). As shown in Supplementary Figure 2, the probe electrodes have a low impedance value at $1 \mathrm{kHz}$ of 790 $\pm 100 \mathrm{k} \Omega$. Note that 16 of the 255 electrodes are non-functional because their impedance value at $1 \mathrm{kHz}$ is higher than $2 \mathrm{M} \Omega$. The noise magnitude computed in saline for the functional electrodes is $5.09 \pm 0.03 \mu \mathrm{V}$. The data shown in Supplementary Figure 3 illustrate the ability of this ultra-highdensity array of electrodes to detect the activity from the same neuron on several adjacent 
electrodes. This dataset can be used to explore the optimal electrode configuration (i.e., electrode size and density) required to record and isolate spikes, which may be specific to different brain regions with different neuron types (i.e., different cell sizes and dendritic configurations) and different neuron densities, and thus require "custom-fit" probes for effective neuron isolation (Buzsáki et al., 2015).

\section{Discussion}

The NeuroSeeker project is the culmination of a number of efforts to translate major advances in microfabrication into new tools for electrophysiology (Jun et al., 2017b; Lopez et al., 2013; Neves et al., 2008; Raducanu et al., 2017; Torfs et al., 2011). The project has produced a device with multiplexing electrode scanning circuitry within the thin shaft of an implantable neural probe, which provides the highest number of simultaneous outputs for a single shaft in vivo extracellular recording device ever achieved.

Here we report that the integrated circuitry of the NeuroSeeker probe does not have an adverse effect on its ability to record neural activity during both acute and chronic recordings. We show that when compared to a passive probe with the same electrode configuration, the NeuroSeeker probe offers simultaneous recordings from $>1000$ electrodes with similar signal-to-noise ratio. We also show that the probe can be used in long term chronic experiments with a signal deterioration over time comparable to other silicon based probes. Finally, we demonstrate that the probe is both mechanically and electrically robust enough to be removed at the end of a chronic experiment and be reused without any loss of signal quality. Given all this, we propose that integrating on-shank scanning circuitry is not only a promising technology for creating ultra-high density in vivo probes, but will, at the very least, allow the simultaneous recording from the entire probe with a density sufficient for accurate spike sorting.

Of course the promise of such high-density, large electrode count probe designs, with their capability to scale to even larger numbers and densities, offers more than simply increasing spike sorting accuracy; they make it possible to simultaneously extract information from the brain on three different scales. At the single neuron level, high densities provide valuable information about cell type and signal propagation throughout the dendritic tree, which can help address the underlying single cell computation. At the level of the local circuit, recording hundreds of neurons simultaneously from a given functional region (here we record from more than 200 per region) is required to test many major hyptoheses in systems neuroscience, e.g. the canonical circuit, the engram, or hebbian learning. Finally, at the level of the global network, recording from a number of regions throughout the brain (here we record from 6,5 of which are subcortical) allows studies of how the brain as a large network of functionally and anatomically diverse regions generates complex behavior. The results we present here demonstrate a new technology that can scale to higher electrode counts with the same, or higher, density of electrodes. Future devices, which distribute dense electrode groups throughout the brain are possible, and clearly worth exploring.

The number of simultaneously recorded electrodes of the NeuroSeeker probe created new challenges for previously simple aspects of an experiment, e.g. online data monitoring. We also present here a GPU-based method for generating a real-time visualization of the very large data sets generated by these probes. This greatly facilitates the control of an ongoing experiment and provided an intuitive overview of large scale structure present in the spiking activity recorded by the probe, which often spans multiple distinct anatomical and functional brain regions. 
Finally, the high costs associated with CMOS development necessitate a large initial investment to produce just a small number of prototype devices. However, the promise of modern CMOS production scaling is that, after an expensive development phase, a large number of equivalent devices can be produced at a tiny fraction of the initial costs. The recent CMOS-based neural probe prototyping efforts, such as NeuroSeeker, have resulted in truly revolutionary devices for neurophysiology. However, a true revolution in neuroscience will only come when these devices are available in large numbers, to the entire community, for the price of a spool of tungsten wire.

\section{Methods}

\section{Animal surgeries}

All experiments were conducted with Lister Hooded rats ranging between $400 \mathrm{~g}$ to $700 \mathrm{~g}$ of both sexes. For both acute and chronic surgeries, the animals were anesthetized, placed in a stereotaxic frame and undergone a surgical procedure to remove the skin and expose the skull above the targeted brain region. For the acute surgeries we used urethane $(1.6 \mathrm{~g} / \mathrm{kg}, \mathrm{IP})$ anesthesia, while for the chronic ones we used isoflurane gas anesthesia $(2 \% \mathrm{v} / \mathrm{v})$. At the initial stage of each surgery the animal was also injected with atropine $(0.05 \mathrm{mg} / \mathrm{kg})$, temgesic $(20 \mu \mathrm{g} / \mathrm{kg}, \mathrm{SC})$ and rimadyl $(5 \mathrm{mg} / \mathrm{kg}$, $\mathrm{SC})$. Small craniotomies (about $1 \mathrm{~mm}$ in diameter) were performed above the target area. During the surgeries equipment for monitoring the animals' body temperature and a video system for guiding probe insertion (Neto et al., 2016) was used. The targeted insertion coordinates were scaled according to the size of each animal's skull. We measured the distance between bregma (B) and interaural line (IA) for all animals to find the ratio between the animal skull and the reference skull (B $-I A=9 \mathrm{~mm}$ ) generated as part of the 3D atlas described in (Dimitriadis et al., 2014). Finally, we adapted the insertion coordinates for targeting specific brain regions with the use of the 3D atlas. Animal experiments were approved by the local ethical review committee and conducted in accordance with Home Office personal and project (xxx; 70/8116) licenses under the UK Animals (Scientific Procedures) 1986 Act.

\section{Recordings}

The CMOS-based probes are assembled on a PCB where the reference (REF) and ground (GND) wires are connected. The recording system consists of a headstage, which configures and calibrates the probe and serializes probe data, a microcoax cable and the base station board with a deserializer chip which connects to a commercial FPGA development board (Xilinx KC705). The computer used for controlling the NeuroSeeker system is connected to the FPGA board via a $1000 \mathrm{kBps}$-T Ethernet card. This recording system together with the FPGA code and low level C drivers for FPGA computer communication were developed by IMEC. The FPGA code and the $C$ drivers remain closed source. The protocol for the communication with the low level $C$ drivers for the acquisition and saving of the data and subsequent visualization was implemented in Bonsai. Both Bonsai and the specific NeuroSeeker communication protocol are open source (Bonsai, 2017; Lopes et al., 2015). The CMOS extracellular signals were sampled at $20 \mathrm{kHz}$ with a 10-bit resolution.

Due to the small diameter of the microcoax cable and the size of the behaviour arena there was no requirement for the use of a commutator during the freely moving animal recordings. No cable tension or significant torsion ever developed during the 1-1.5 hour long experimental sessions. The behaviour arena is a $1 \times 1 \mathrm{~m}$ box with a back projection glass surface as floor onto which a computer projects imagery at a frame rate of $120 \mathrm{~Hz}$. A grasshopper 3 (FLIR) camera is recording the 
movements of the rat in the arena, also at $120 \mathrm{~Hz}$, while the controlling computer system is live tracking the animal generating $x$, y positions for each frame.

For the 256-channel probe recordings we used the Open Ephys (http://www.open-ephys.org/) acquisition board along with two RHD2000 128-channel amplifier boards that amplify and digitally multiplex the extracellular electrodes (Intan Technologies). Extracellular signals in a frequency band of $0.1-7,500 \mathrm{~Hz}$ were sampled at $20 \mathrm{kHz}$ with 16-bit resolution and were saved in a raw binary format for subsequent offline analysis using Bonsai.

\section{Impedance measurements}

For the 256-channel probe, impedance tests (at $1 \mathrm{kHz}$ ) were performed using a protocol implemented by the RHD2000 series chip (Intan Technologies) with the electrodes placed in a dish with sterile PBS, $1 \mathrm{mM}, \mathrm{pH} 7.4$ and a reference electrode, $\mathrm{Ag}-\mathrm{AgCl}$ wire (Science Products $\mathrm{GmbH}, \mathrm{E}$ 255).

\section{Probe removal}

The probe removal from a chronic implantation starts by aligning the rat's head (after euthanasia) with the stereotactic coordinates system. This was done by making sure the probe holder's top surface is parallel to the Anterior-Posterior (AP) / Medial-Lateral (ML) plane of the stereotaxic frame. To achieve this, we make sure this plane is perpendicular to gravity using a spirit level. We then make sure the same thing is true for the rat's head in the stereotaxic frame, i.e. the top surface of the probe's holder is also perpendicular to gravity (using again a spirit level). This step follows from the fact that during probe implantation the probe was held by the stereotaxic frame (while glued on the rat's skull) in such a way that it's top surface was parallel to the frame's AP/ML plane, so by realigning those two planes we bring the probe back to the original position it was implanted in respect to the Dorsal-Ventral (DV) movement of the stereotaxic frame's arm. After alignment, we connect the probe holder to the frame's arm, and then cut through the thin layer of resin (that is kept away from the probe by the tapered skirt), thus separating the part that stays attached onto the head and the part that holds the probe. Then we slowly remove the detached probe by withdrawing the stereotaxic frame's arm. The alignment of the head and the connection of the arm to the still attached probe holder is of importance since any tension between the head and the frame's arm due to misalignment will be released after the cutting of the resin in the form of a movement of the head in respect to the arm resulting in the breaking of the probe shaft. After removal a series of trypsin and $\mathrm{dd}_{2} \mathrm{O}$ cleaning cycles were undertaken until, under a microscope, no organic or salt residues could be seen on the probe's shaft.

\section{Analysis}

For the analysis, the CMOS-based probe recordings were filtered with a band-pass of $500-3,500 \mathrm{~Hz}$ and they were saved in binary format using a Bonsai interface. When the external reference was selected, to diminish the effect of artefacts shared across all channels, we subtracted the median signal within each group across the recording electrodes from the respective group.

For the reconstruction of a unit's position on the probe (for the results shown in Figure 3 ) we used a weighted (according to peak to peak amplitude (PPA)) average of the positions of the electrodes that showed a significant deviation from baseline on the average spike waveform. That is, we selected 
the electrode with the highest PPA at the time around 0 of a unit's spike template, found all other electrodes whose PPA was over a threshold and then moved the position of the unit from the highest electrode's position towards the other relevant electrodes in a linearly weighted manner according to their PPAs.

For the 256-channel probes, a third order Butterworth filter with a band-pass of $250-9,500 \mathrm{~Hz}$ (95\% of the Nyquist frequency) was used in the forward-backward mode. The noise magnitude was computed as an estimate of the background noise, $\sigma_{\text {Median }}=$ median $(|\operatorname{signal}(\mathrm{t})| / 0.6745)$, of each filtered voltage-time trace during 5 seconds. Some results were represented as mean \pm standard deviation.

\section{Acknowledgments}

Joana Neto was supported by the fellowship SFRH/BD/76004/2011 from Fundação para a Ciência e Tecnologia, Portugal.

This work was supported by funding from the European Union's Seventh Framework Programme (FP7/2007-2013) under grant agreement nr. 600925 (NeuroSeeker), and by the the Bial Foundation (Grant 190/12).

\section{References}

Berényi, A., Somogyvári, Z., Nagy, A.J., Roux, L., Long, J.D., Fujisawa, S., Stark, E., Leonardo, A., Harris, T.D., and Buzsáki, G. (2014). Large-scale, high-density (up to 512 channels) recording of local circuits in behaving animals. J. Neurophysiol. 111, 1132-1149.

Blanche, T.J. (2005). Polytrodes: High-Density Silicon Electrode Arrays for Large-Scale Multiunit Recording. J. Neurophysiol. 93, 2987-3000.

Buzsáki, G., Stark, E., Berényi, A., Khodagholy, D., Kipke, D.R., Yoon, E., and Wise, K.D. (2015). Tools for Probing Local Circuits: High-Density Silicon Probes Combined with Optogenetics. Neuron 86, 92105.

Delgado Ruz, I., and Schultz, S.R. (2014). Localising and classifying neurons from high density MEA recordings. J. Neurosci. Methods 233, 115-128.

Dimitriadis, G., Fransen, A.M.M., and Maris, E. (2014). Sensory and cognitive neurophysiology in rats, Part 1: Controlled tactile stimulation and micro-ECoG recordings in freely moving animals. J. Neurosci. Methods 232, 63-73.

Dimitriadis, G., Neto, J., and Kampff, A. (2016). T-SNE visualization of large-scale neural recordings.

Du, J., Blanche, T.J., Harrison, R.R., Lester, H.A., and Masmanidis, S.C. (2011). Multiplexed, High Density Electrophysiology with Nanofabricated Neural Probes. PLoS ONE 6, e26204.

Jia, X., Siegle, J., Bennett, C., Gale, S., Denman, D., Koch, C., and Olsen, S. (2018). High-density extracellular probes reveal dendritic backpropagation and facilitate neuron classification. 
Jun, J., Mitelut, C., Lai, C., Gratiy, S.L., Anastassiou, C.A., and Harris, T.D. (2017a). Real-time spike sorting platform for high-density extracellular probes with ground-truth validation and drift correction. BioRxiv 1-29.

Jun, J.J., Steinmetz, N.A., Siegle, J.H., Denman, D.J., Bauza, M., Barbarits, B., Lee, A.K., Anastassiou, C.A., Andrei, A., Aydın, Ç., et al. (2017b). Fully integrated silicon probes for high-density recording of neural activity. Nature 551, 232-236.

Lopes, G., Bonacchi, N., Frazão, J., Neto, J.P., Atallah, B. V, Soares, S., Moreira, L., Matias, S., Itskov, P.M., Correia, P.A., et al. (2015). Bonsai: an event-based framework for processing and controlling data streams. Front. Neuroinformatics 9, 7.

Lopez, C.M., Andrei, A., Mitra, S., Welkenhuysen, M., Eberle, W., Bartic, C., Puers, R., Yazicioglu, R.F., and Gielen, G. (2013). An implantable 455-active-electrode 52-channel CMOS neural probe. In 2013 IEEE International Solid-State Circuits Conference Digest of Technical Papers, (IEEE), pp. 288-289.

Lopez, C.M., Mitra, S., Putzeys, J., Raducanu, B., Ballini, M., Andrei, A., Severi, S., Welkenhuysen, M., Van Hoof, C., Musa, S., et al. (2016). A 966-electrode neural probe with 384 configurable channels in $0.13 \mu \mathrm{m}$ SOI CMOS. In 2016 IEEE International Solid-State Circuits Conference (ISSCC), (IEEE), pp. 392-393.

Moore-Kochlacs, C.E. (2016). Extracellular electrophysiology with close-packed recording sites: spike sorting and characterization. Boston University.

Najafi, K., and Wise, K.D. (1986). An implantable multielectrode array with on-chip signal processing. IEEE J. Solid-State Circuits 21, 1035-1044.

Najafi, K., Wise, K.D., and Mochizuki, T. (1985). A high-yield IC-compatible multichannel recording array. IEEE Trans. Electron Devices 32, 1206-1211.

Neto, J.P., Lopes, G., Frazão, J., Nogueira, J., Lacerda, P., Baião, P., Aarts, A., Andrei, A., Musa, S., Fortunato, E., et al. (2016). Validating silicon polytrodes with paired juxtacellular recordings: method and dataset. J. Neurophysiol. 116, 892-903.

Neves, H.P., Torfs, T., Yazicioglu, R.F., Aslam, J., Aarts, A.A., Merken, P., Ruther, P., and Van Hoof, C. (2008). The NeuroProbes project: a concept for electronic depth control. Conf. Proc. Annu. Int. Conf. IEEE Eng. Med. Biol. Soc. IEEE Eng. Med. Biol. Soc. Annu. Conf. 2008, 1857.

Obien, M.E.J., Deligkaris, K., Bullmann, T., Bakkum, D.J., and Frey, U. (2015). Revealing neuronal function through microelectrode array recordings. Front. Neurosci. 8, 423.

Pachitariu, M., Steinmetz, N., Kadir, S., Carandini, M., and Harris, K.D. (2016). Kilosort: realtime spike-sorting for extracellular electrophysiology with hundreds of channels.

Raducanu, B.C., Yazicioglu, R.F., Lopez, C.M., Ballini, M., Putzeys, J., Wang, S., Andrei, A., Welkenhuysen, M., Van Helleputte, N., Musa, S., et al. (2016). Time multiplexed active neural probe with 678 parallel recording sites. In European Solid-State Device Research Conference, (IEEE), pp. 385-388.

Raducanu, B.C., Yazicioglu, R.F., Lopez, C.M., Ballini, M., Putzeys, J., Wang, S., Andrei, A., Rochus, V., Welkenhuysen, M., Helleputte, N. van, et al. (2017). Time Multiplexed Active Neural Probe with 1356 Parallel Recording Sites. Sensors 17, 2388. 
Rios, G., Lubenov, E. V., Chi, D., Roukes, M.L., and Siapas, A.G. (2016). Nanofabricated Neural Probes for Dense 3-D Recordings of Brain Activity. Nano Lett. 16, 6857-6862.

Rossant, C., Kadir, S.N., Goodman, D.F.M., Schulman, J., Belluscio, M., Buzsaki, G., and Harris, K.D. (2015). Spike sorting for large, dense electrode arrays (Cold Spring Harbor Labs Journals).

Ruther, P., and Paul, O. (2015). New approaches for CMOS-based devices for large-scale neural recording. Curr. Opin. Neurobiol. 32, 31-37.

Scholten, K., and Meng, E. (2016). Electron-beam lithography for polymer bioMEMS with submicron features. Microsyst. Nanoeng. 2, 16053.

Scholvin, J., Kinney, J.P., Bernstein, J.G., Moore-Kochlacs, C., Kopell, N., Fonstad, C.G., and Boyden, E.S. (2016). Close-packed silicon microelectrodes for scalable spatially oversampled neural recording. IEEE Trans. Biomed. Eng. 63, 120-130.

Seymour, J.P., Wu, F., Wise, K.D., and Yoon, E. (2017). State-of-the-art MEMS and microsystem tools for brain research. Microsyst. Nanoeng. 3, 16066.

Shobe, J.L., Claar, L.D., Parhami, S., Bakhurin, K.I., and Masmanidis, S.C. (2015). Brain activity mapping at multiple scales with silicon microprobes containing 1024 electrodes. J. Neurophysiol. 114, 2043-2052.

Steinmetz, N.A., Koch, C., Harris, K.D., and Carandini, M. (2018). Challenges and opportunities for large-scale electrophysiology with Neuropixels probes. Curr. Opin. Neurobiol. 50, 92-100.

Stevenson, I. (2017). Tracking Advances in Neural Recording.

Stevenson, I.H., and Kording, K.P. (2011). How advances in neural recording affect data analysis. Nat. Neurosci. 14, 139-142.

Torfs, T., Aarts, A. a. A., Erismis, M.A., Aslam, J., Yazicioglu, R.F., Seidl, K., Herwik, S., Ulbert, I., Dombovari, B., Fiath, R., et al. (2011). Two-dimensional multi-channel neural probes with electronic depth control. IEEE Trans. Biomed. Circuits Syst. 5, 403-412.

Wise, D.K., and Ji, J. (1992). An Implantable CMOS Circuit Interface for Multiplexed Microelectrode Recording Arrays. IEEE J. Solid-State Circuits 27, 433-443.

Wise, K.D., and Najafi, K. (1991). Microfabrication techniques for integrated sensors and microsystems. Science 254, 1335-1342. 


\section{Box 1. NeuroSeeker CMOS-based probe.}

The NeuroSeeker probe contains 1344 small electrodes $(20 \times 20 \mu \mathrm{m})$ with $2.5 \mu \mathrm{m}$ spacing and 12 larger reference electrodes $(40 \times 80 \mu \mathrm{m})$.

Time division multiplexing within the shank allows 8 electrode outputs on a single output connection. If a single metal line can be shared by several electrodes, we are no longer restrained by the number of metal lines that can fit within the cross-section of a shank or by the size of the bonding interface in the probe base.

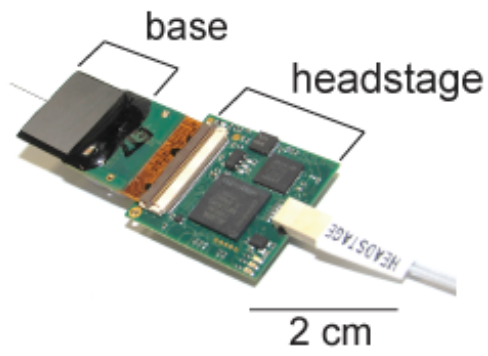

The challenge in such a design is fitting all the required electronics within the electrode area, while making sure the power dissipation is sufficiently low for tissue safety. Due to the high impedance of the electrode, each requires its own amplifier to drive the multiplexed output line (Obien et al., 2015).

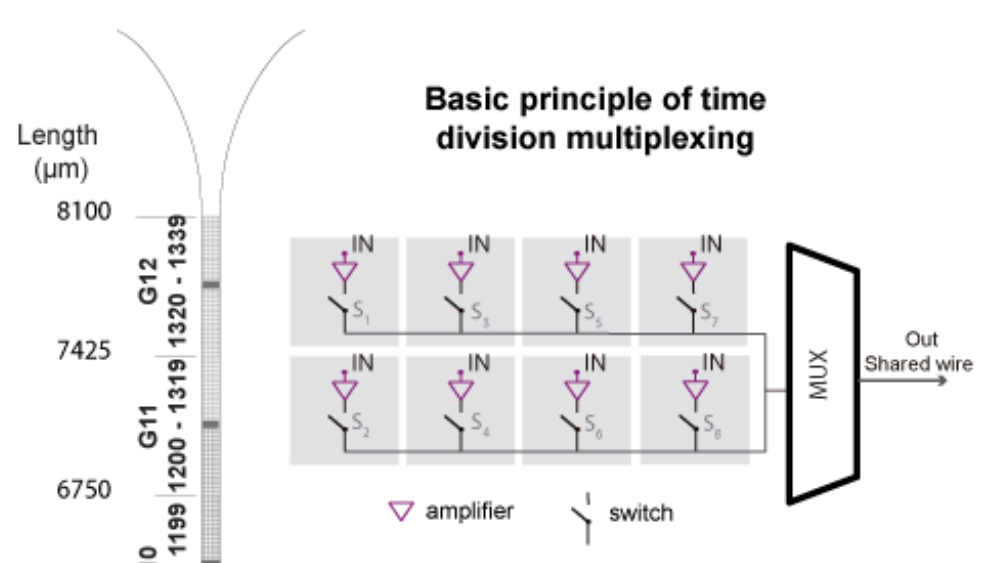

6075

For each electrode the input voltage (IN) is converted to an output current which is integrated for a fixed period of time $(\mathrm{Ti}=2.5$ $\mu \mathrm{s})$ over a capacitor $(\mathrm{Ci}=15 \mathrm{pF})$ shared among 8 electrodes in the probe base. After $\mathrm{Ti}$, the voltage on $\mathrm{Ci}$ is sampled and then it is discharged for the next cycle. The integration reduces out-of-band noise acting as anti-aliasing filter. Further noise reduction is possible by oversampling each electrode at fs $=40 \mathrm{kHz}$ (neural signal band is limited to $\sim 7.5 \mathrm{kHz}$, therefore fs $>15 \mathrm{kHz}$ ), wich produces a total multiplexing frequency of $320 \mathrm{kHz}$. The signal is fed to an integrator, in the base, whose output is demultiplexed (DMUX block).

3375

2700

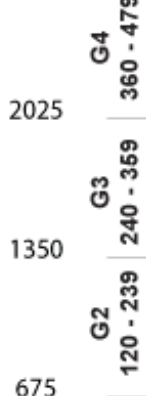

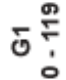

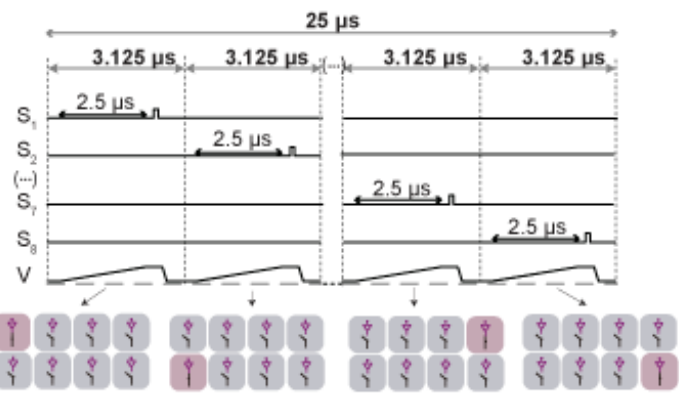

Each reference electrode is made from a bin of 8 electrodes, therefore the circuit handles, in total, 1440 channels. A total of 180 Integrator-DMUX blocks drive the 1440 channels, which are further amplified and filtered on the base, keeping only the band of interest. These signals are then multiplexed and digitized in groups of 20 channels (10-bit successive approximation analog-to-digital converter) by 72 ADCs Finally, a digital control block is responsible for generating the clocks for the ADCs and the MUX/DMUX blocks. It also serializes the parallel data from all the ADCs to only 6 data lines (Raducanu et al., 2017). 
A

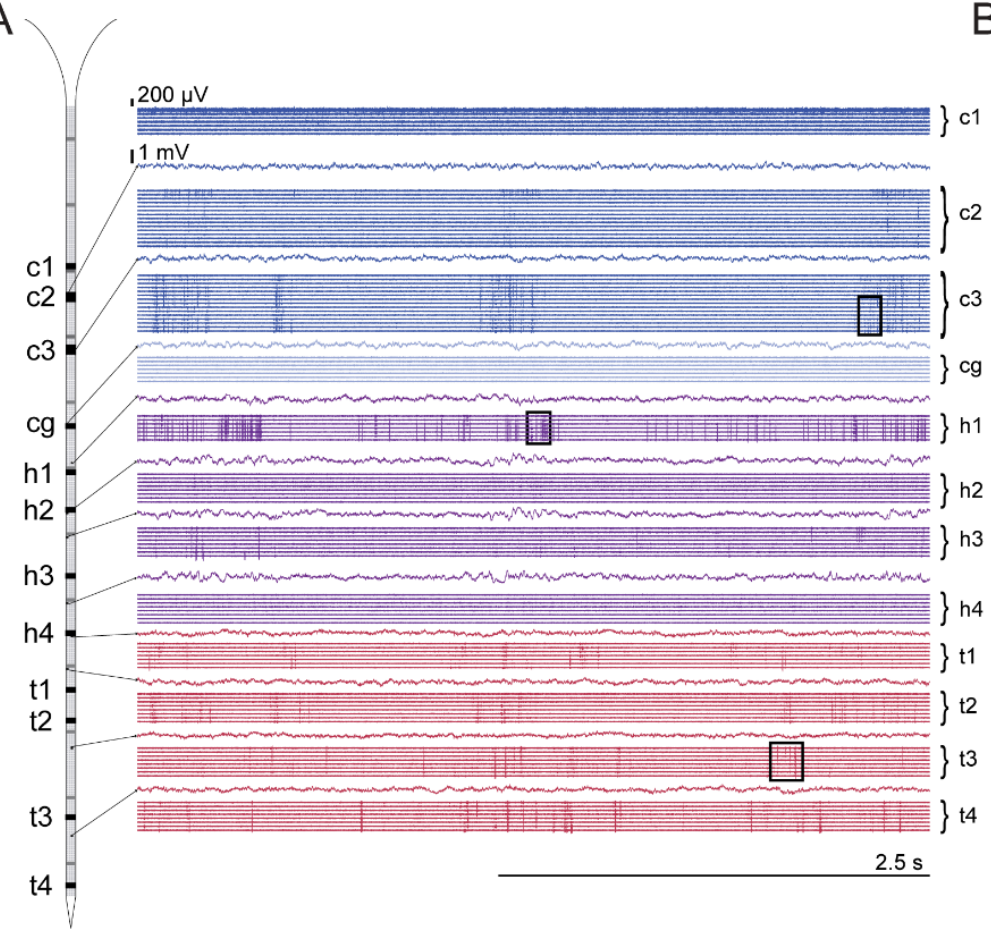

C

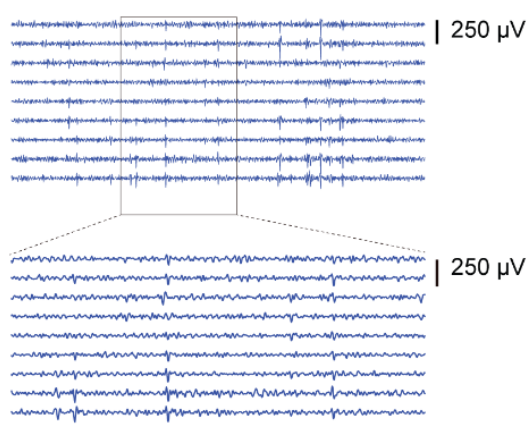

B
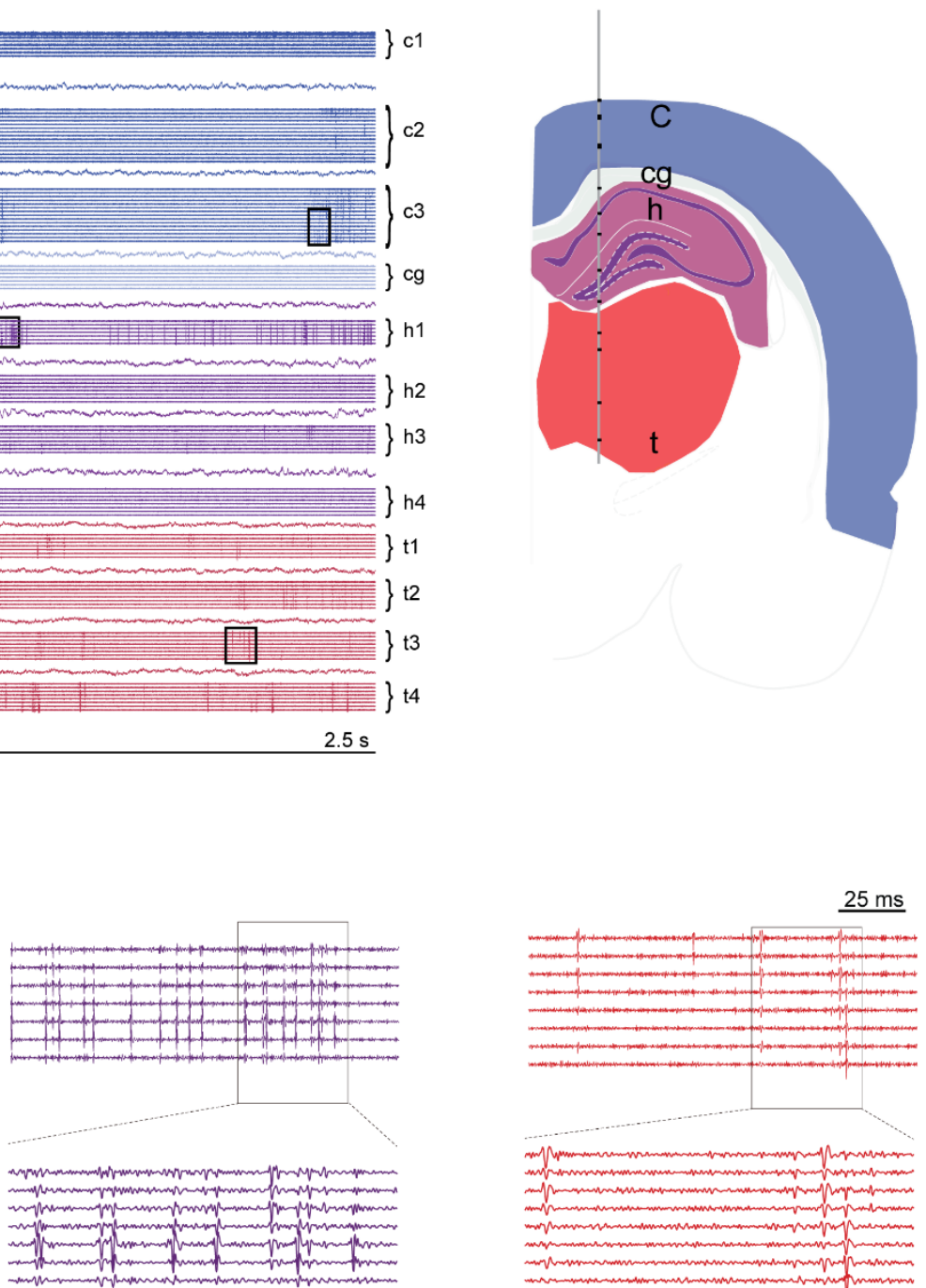

$\underline{25 \mathrm{~ms}}$

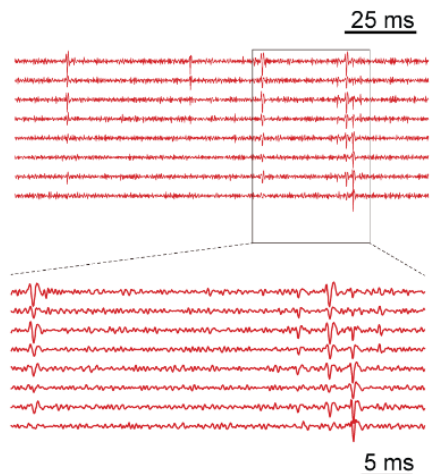

Figure 1. Example of a recording performed with 1120 electrodes simultaneously within cortex, hippocampus, and thalamus from an anesthetized rat. A) 5-s-long LFP and AP traces of a probe spanning multiple brain regions; B) Schematic of coronal slice signalling the group's position shown in A as black blocks. 'c, 'cg', ' $h$ ' and ' $t$ ' denote the anatomic locations of cortex, cingulum, hippocampus, and thalamus, respectively. The estimated probe position was based on the insertion coordinates and physiological signatures. From the point of insertion to the tip, the recording depth was $6.7 \mathrm{~mm}$; C) Short epochs of AP band traces from groups c3, h1, and t3 (color-coded), illustrating the presence of diverse spike waveforms on several electrodes. These sections are highlighted in A by boxed areas on top of traces. 

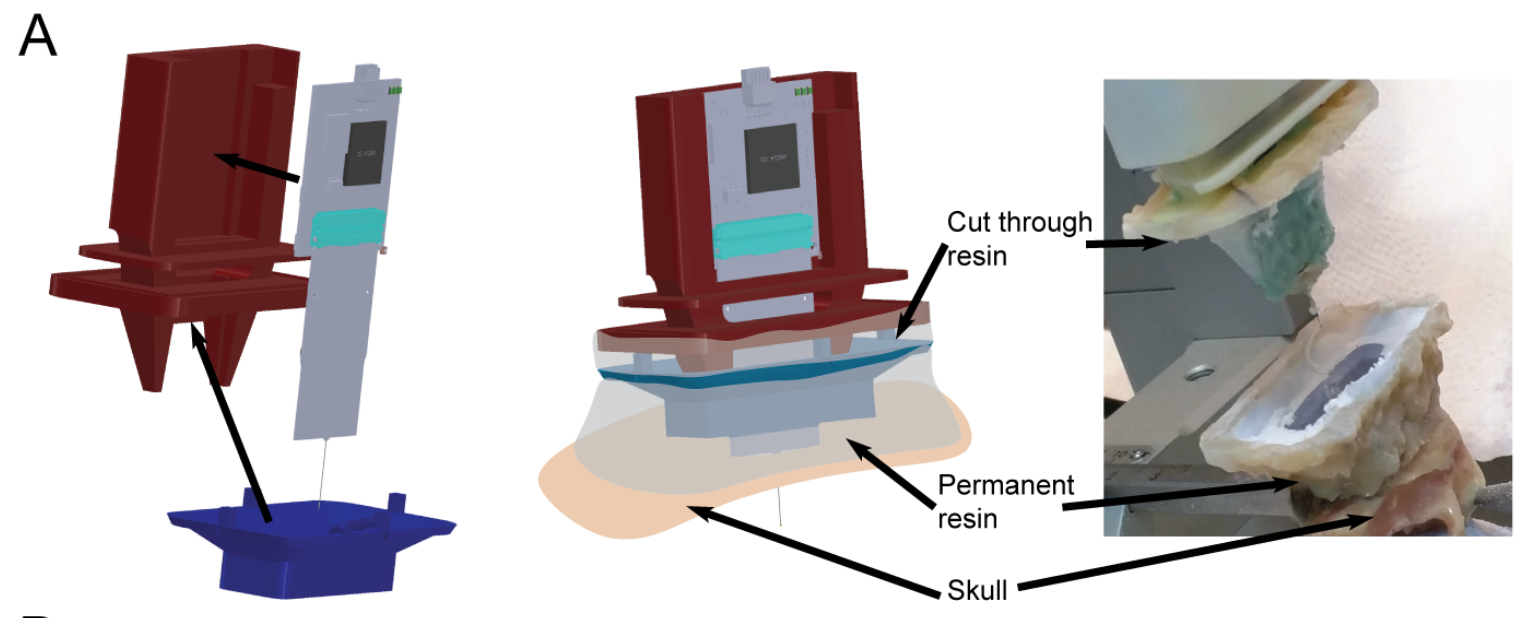

B
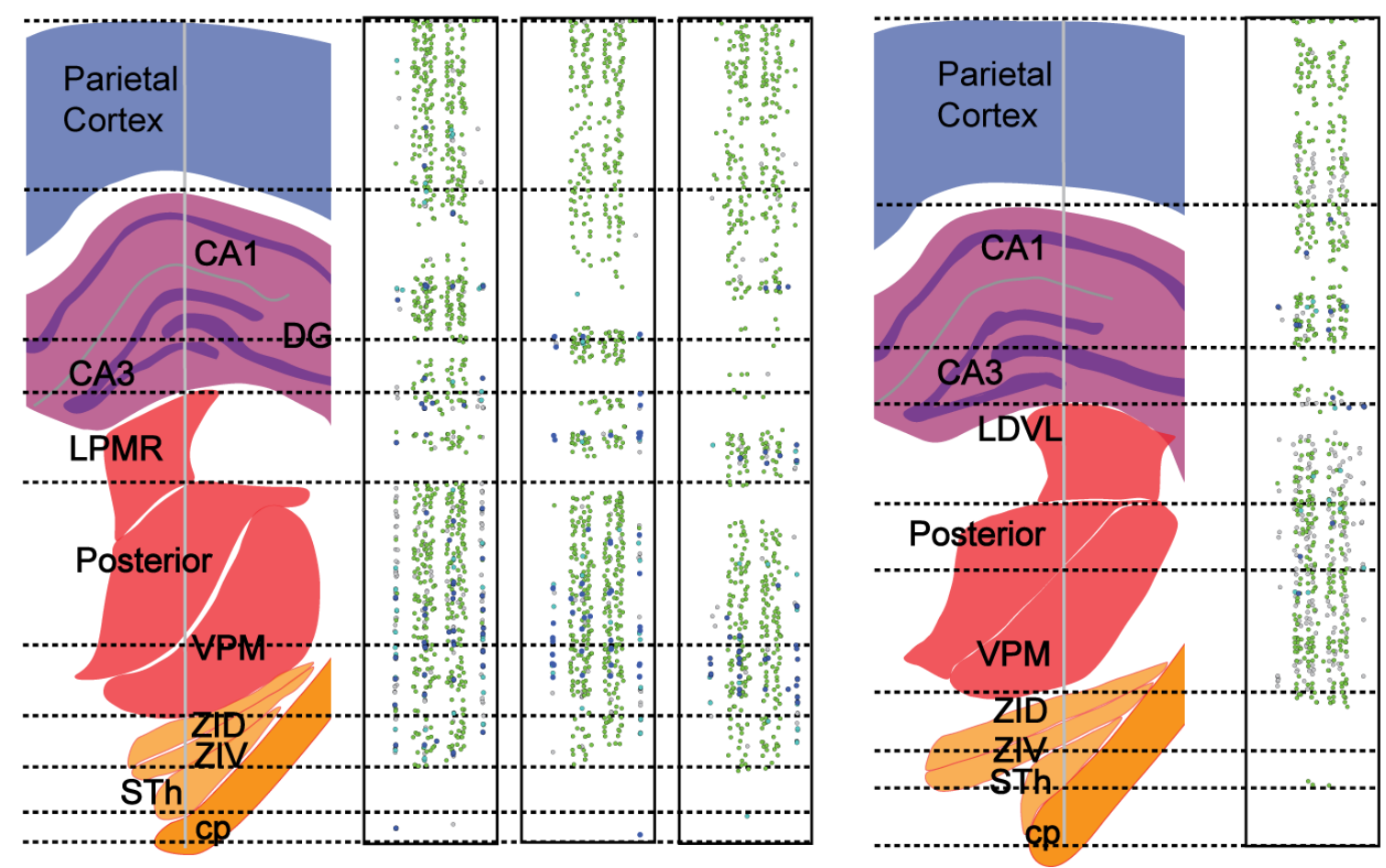

C

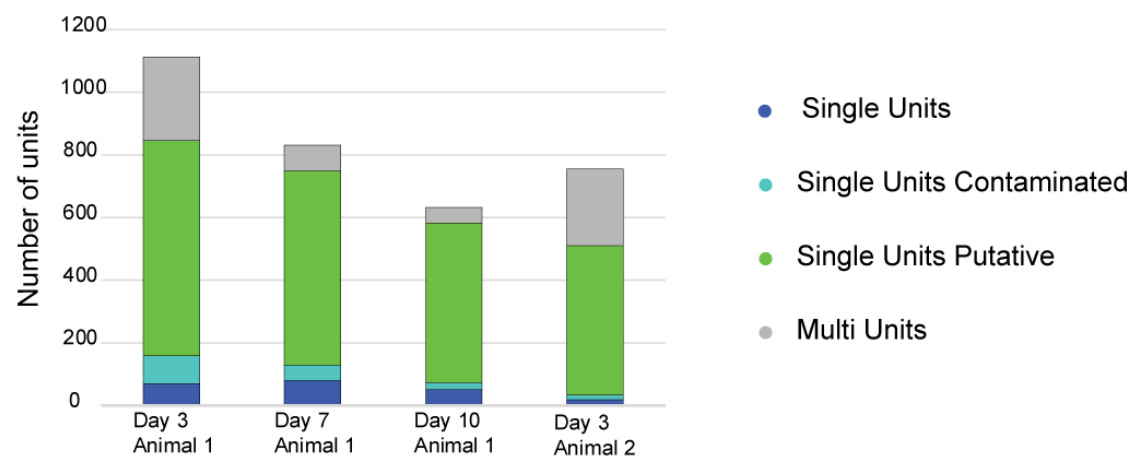

Figure 2. Chronic recordings and probe removal process. A) The processes of inserting the probe before surgery (left), implanting and gluing the probe in place during surgery (middle) and removing the probe by cutting the cut-through resin layer after surgery (right). B) Results from two animals chronically implanted with the same probe (left animal first, right animal second). The two anatomy 
schematics show where the probe was implanted in each animal. The scatter plots show the position of the recorded units on the probe for one recording session. Each dot is a unit (dark blue, light blue and green), or a multi unit group (grey) and the axis are the $y$ and $x$ coordinates of the probe's shaft (not shown to the same scale due to the extremely elongated nature of the shaft). The three position plots of the first animal show recordings done 3, 7 and 10 days after surgery, while the plot for the second animal show the first recording, done on the $3^{\text {rd }}$ day after surgery. C) Cumulative bar chart showing the number of recorded units over days and animals. Brain regions: CA1: Cornu Ammonis 1, CA3: Cornu Ammonis 3, DG: Dentate Gyrus, LPMR: Lateral Posterior MedioRostral thalamic nucleus, LDVL: Lateral Dorsal VentroLateral thalamic nucleus: VPM: Ventral Posterior Medial thalamic nucleus, ZID: Zona Incerta Dorsal, ZIV: Zona Incerta Ventral, Sth: SubThalamic nuclei, cp: cerebral penducle 


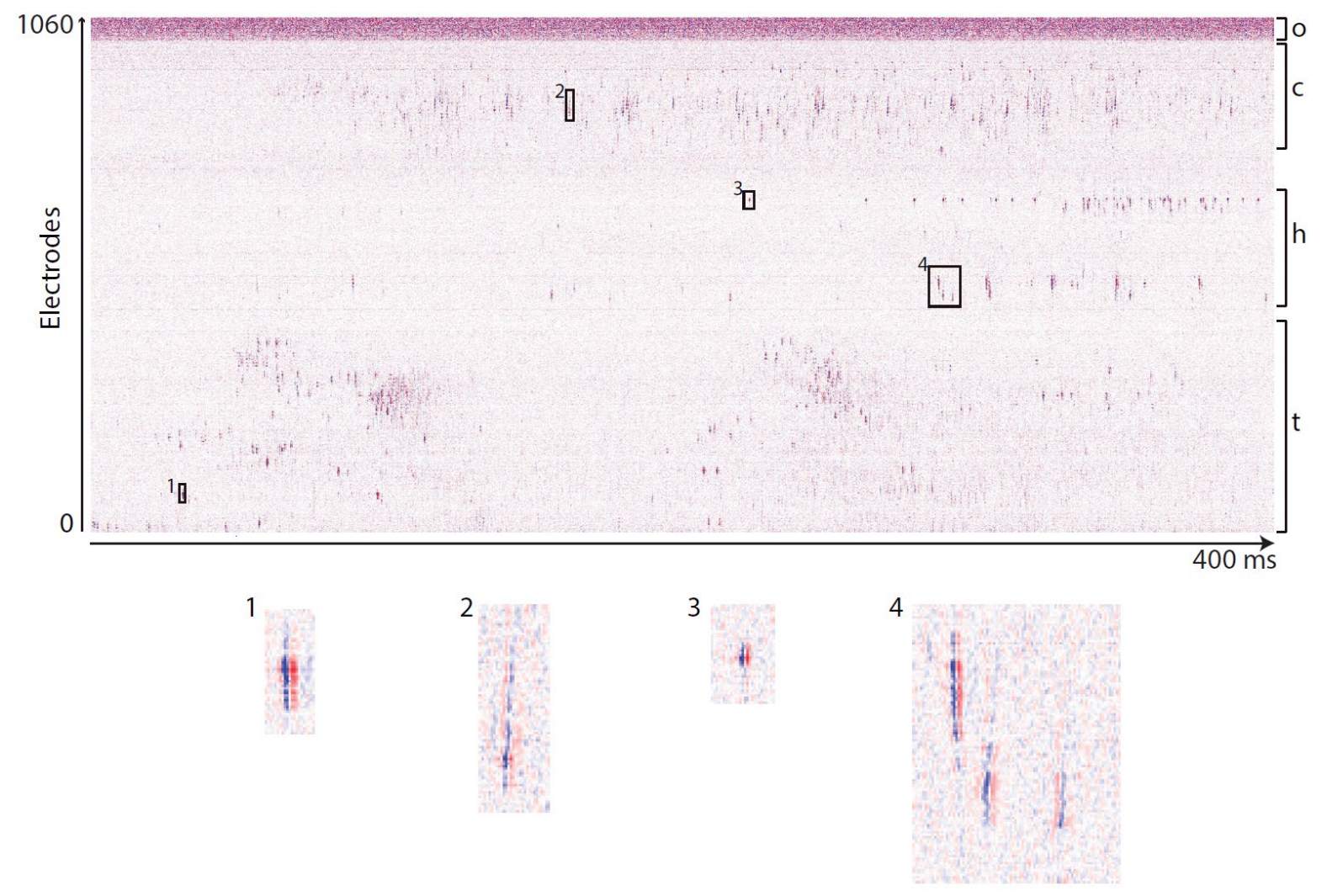

Figure 3. Real time GPU-based visualization of the voltage traces from 1060 electrodes in AP mode during acute recordings under anesthesia. The voltage traces recorded by each electrode (row) are displayed as a color saturation for each 100 microsecond bin (column). Red represents positive voltages (maximum color value represents $150 \mu \mathrm{V}$ ), blue negative voltages (minimum color value represents $-150 \mu \mathrm{V}$ ), and white represents voltages near zero. This image shows 400 milliseconds of data recorded simultaneously in cortex, hippocampus and thalamus. ' $\mathrm{o}$ ': out of the brain, ' $c$ ': cortex, ' $h$ ': hippocampus and ' $t$ ': thalamus. 
CMOS scanning probe

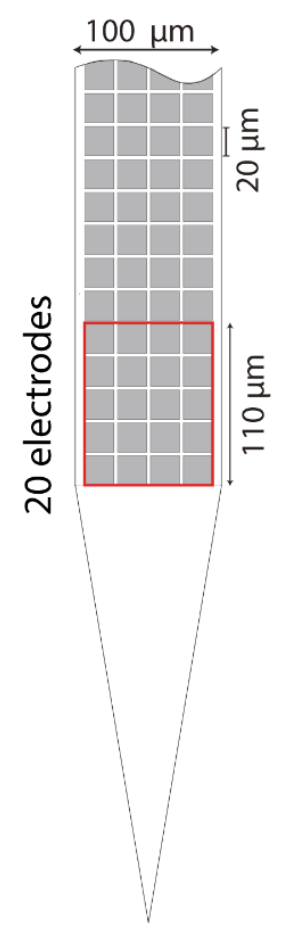

256-channel probe

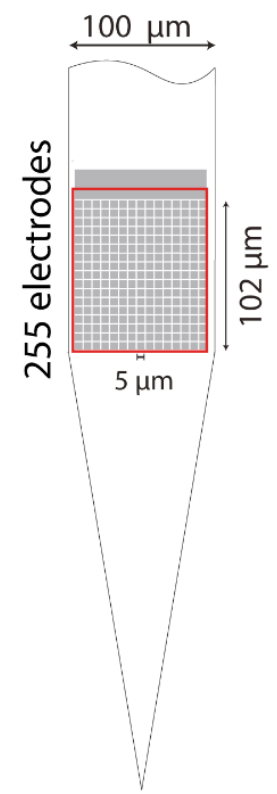

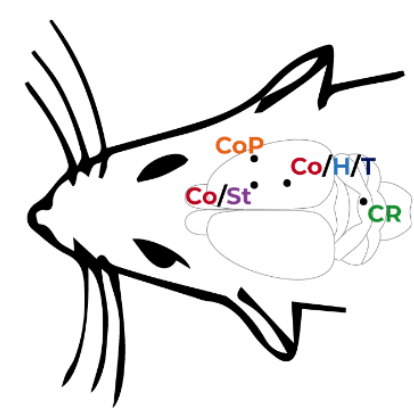

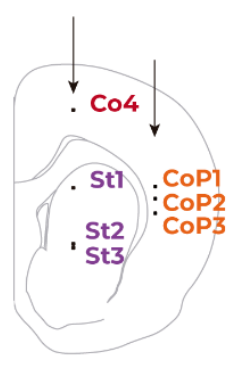

Figure 4. The 256-channel ultra-dense probe packs 255 electrodes in the same area $(100 \times 102 \mu \mathrm{m})$ as 20 electrodes from the NeuroSeeker CMOS scanning probe, a 13x increase in density of electrodes. We conducted four separate penetrations covering five anatomically distinct brain regions and two different orientations (i.e., parallel and perpendicular) with respect to the laminar architecture of cortex, leading to 18 separate recordings.

\section{Brain Region}

Cortex (II to the layers) Cortex ( $\perp$ to the layers) Cerebellum

Hippocampus

Thalamus

Striatum

\section{Recording Labels}

$\mathrm{Co} 1, \mathrm{Co} 2, \mathrm{Co} 3, \mathrm{Co} 4$

COP1, COP2, COP3

CR1, CR2, CR3

$\mathrm{H} 1, \mathrm{H} 2, \mathrm{H} 3$

$\mathrm{T}, \mathrm{T} 2$

St1, St2, St3 
bioRxiv preprint doi: https://doi.org/10.1101/275818; this version posted February 9, 2019. The copyright holder for this preprint (which was not certified by peer review) is the author/funder, who has granted bioRxiv a license to display the preprint in perpetuity. It is made available under aCC-BY 4.0 International license.

\begin{tabular}{|l|l|l|l|l|}
\hline & $1^{\text {st }}$ Animal / Day 3 & $1^{\text {st }}$ Animal / Day 7 & $1^{\text {st }}$ Animal / Day 10 & $2^{\text {nd }}$ Animal / Day 3 \\
\hline Single Units & 70 & 80 & 51 & 19 \\
\hline $\begin{array}{l}\text { Single Units } \\
\text { Contaminated }\end{array}$ & 89 & 48 & 22 & 14 \\
\hline $\begin{array}{l}\text { Single Units } \\
\text { Putative }\end{array}$ & 687 & 622 & 509 & 477 \\
\hline Multi Units & 267 & 81 & 51 & 246 \\
\hline Total Units & 1113 & 831 & 633 & 756 \\
\hline Spikes & $3,586,965$ & $2,610,528$ & $2,536,517$ & $1,449,147$ \\
\hline Recording Time & $40: 30$ & $46: 58$ & $1: 00: 05$ & $49: 28$ \\
\hline
\end{tabular}

Table 1. A summary of the number of units and number of spikes recorded during four representative sessions 